\title{
Screening differential circular RNA expression profiles reveal that hsa_circ_0I 28298 is a biomarker in the diagnosis and prognosis of hepatocellular carcinoma
}

This article was published in the following Dove Press journal:

Cancer Management and Research

\author{
Dawei Chen ${ }^{1,2, *}$ \\ Chenyue Zhang ${ }^{3, *}$ \\ Jiamao $\operatorname{Lin}^{4}$ \\ Xinyu Song 2,4 \\ Haiyong Wang ${ }^{4}$
}

'Department of Radiation Oncology, Shandong Cancer Hospital Affiliated to Shandong University, ${ }^{2}$ School of Medicine and Life Sciences, University of Jinan-Shandong Academy of Medical Sciences, Jinan, ${ }^{3}$ Department of Integrative Oncology, Fudan University Shanghai Cancer Center, Shanghai, ${ }^{4}$ Department of Internal Medicine-Oncology, Shandong Cancer Hospital Affiliated to Shandong University, Shandong Academy of Medical Sciences, Jinan, People's Republic of China

*These authors contributed equally to this work
Correspondence: Haiyong Wang Department of Internal MedicineOncology, Shandong Cancer Hospital and Institute, Shandong Cancer Hospital Affiliated to Shandong University, Shandong Academy of Medical Sciences, Jiyan Road 440, Jinan, Shandong 250II7, People's Republic of China $\mathrm{Tel}+86531676261 \mathrm{II}$

Email wanghaiyong6688@I26.com
Aim: The aim of this study was to analyze the diagnostic and prognostic values of the circular RNA (circRNA) hsa_circ_0128298 in hepatocellular carcinoma (HCC).

Patients and methods: The global circRNA expression was measured using circRNA microarray using three pairs of cancer and noncancerous tissues from HCC patients. The microarray analysis revealed that two circRNAs were differentially expressed in the three pairs of cancerous and noncancerous tissues. The higher levels of two representative circRNAs, such as hsa_circ_0128298 and hsa_circ_0091582, were further confirmed by real-time polymerase chain reaction. In addition, the association between the expression level of hsa_circ_0128298 and the clinicopathological features of patients with HCC was further analyzed. The clinical diagnosis value was confirmed by receiver operating characteristic (ROC) curve analysis. Independent prognostic factors of patient outcome were identified using the Cox regression model. The survival data were analyzed by the Kaplan-Meier method, and the differences were evaluated using log-rank tests. Two-sided $P$-values $<0.05$ were considered statistically significant.

Results: The expression levels of hsa_circ_0128298 in HCC were significantly higher than those of paratumorous tissues $(P<0.001)$. Additionally, hsa_circ_0128298 was a diagnostic factor, with the area under the ROC curve of $0.668(95 \% \mathrm{CI}=0.503-0.794, P<0.001)$. The sensitivity and specificity values were 0.716 and 0.815 , respectively. The AFP and hsa_circ_0128298 expression levels were independent prognostic factors. The overall survival of patients with low hsa_circ_0128298 expression was significantly higher than that of patients with high hsa_circ_0128298 expression.

Conclusion: hsa_circ_0128298 may promote proliferation and metastasis and potentially represents a novel diagnostic and prognostic biomarker for HCC patients. However, studies with larger sample size are needed to confirm our conclusion.

Keywords: hepatocellular carcinoma, circular RNA, hsa_circ_0128298, biomarker, diagnosis, prognosis

\section{Introduction}

Hepatocellular carcinoma (HCC) is one of the most common malignancies in the world and ranks third in the number of cancer-related deaths. ${ }^{1,2}$ Currently, surgical resection remains the primary option for patients with resectable $\mathrm{HCC}$, but the rate of tumor recurrence remains high even after operation due to the refractory property of tumors. ${ }^{3}$ Therefore, treatments to combat HCC recurrence and metastasis are prioritized. Some 
strategies have been adopted to suppress HCC metastasis, but they did not show obvious positive effects. ${ }^{4}$ Therefore, exploring molecular mechanisms and new therapeutic targets is crucial for treating HCC. Unfortunately, there is no clinical tumor marker that provides both satisfactory sensitivity and specificity in the diagnosis and prognosis of HCC. Therefore, it is necessary to study the molecular mechanisms underlying the occurrence and development of HCC and to explore the tumor markers that can ensure an early diagnosis of liver cancer.

Circular RNA (circRNA) is a type of noncoding RNA that exists widely in mammals and is mainly involved in gene regulation in vivo. ${ }^{5-7}$ Most circRNAs are derived from the exon regions of the genes, and a small percentage is spliced by introns. ${ }^{8,9}$ They differ from long noncoding RNA (lncRNA) and microRNAs (miRNAs) in that they do not have the $5^{\prime}$ and $3^{\prime}$ end structures but represent covalently closed cyclic structures. ${ }^{10}$ CircRNA is widely involved in the regulation of human physiology and pathology by the following three main mechanisms: 1) as an miRNA "sponge" (miRNA sponge), 2) as a protein-binding molecule, and 3) as a template for translation into polypeptides. The mechanisms of miRNA sponge regulation of downstream target genes have been widely reported. ${ }^{11-13}$

Since the global circRNA expression profile in HCC has not been widely studied, in our study, we tested the circRNA expression profile in a cohort of HCC patients. We identified differentially expressed circRNAs in HCC tissues compared with those in paratumorous tissues. hsa_circ_0128298, which is transcribed from ENST00000296695 on chromosome 5, has never been studied before (fold change $=127.67, P$-value $=0.006$ ). Therefore, we focused on its association with both the clinicopathological features and prognosis of HCC.

\section{Patients and methods}

\section{Patients and samples}

$\mathrm{HCC}$ and paratumor tissues were collected from patients with HCC who underwent surgery at Fudan University Shanghai Cancer Center between January 1, 2011, and December 31, 2015. Informed consent was obtained from the patients before sample collection, in line with the institutional guidelines. The paratumor samples were collected from tissues $3 \mathrm{~cm}$ from the edge of the HCC to guarantee the absence of tumor cells. These tissue specimens were immediately placed in RNA fixer reagent and kept at $-80^{\circ} \mathrm{C}$. Histology was independently assessed by two experienced pathologists who were blinded to the clinical data.

\section{Ethical approval}

The study was approved by the Ethics Committee of Shandong Cancer Hospital affiliated with Shandong University. All procedures performed in the studies involving human participants were in accordance with the ethical standards of the institutional and/or national research committee and with the 1964 Declaration of Helsinki and its later amendments or comparable ethical standards. Written informed consent was obtained from all patients before their participation in this clinical research.

\section{RNA isolation, reverse transcription (RT), and quantitative real-time polymerase chain reaction ( $q R T-P C R$ )}

Total RNA from all tissues was extracted by using the TRIzol Reagent (Thermo Fisher Scientific, Waltham, MA, USA). All complementary DNAs (cDNAs) were obtained with the GoScriptÔ Reverse Transcription System (Promega Corporation, Fitchburg, WI, USA) following the manufacturer's instructions. The sequences of hsa_circ_0128298 divergent primers were as follows: forward, 5'-TAGACTCAACAGGGCCAAGG-3'; reverse, 5'-TCCATCTTTCTTGAGCAGCA-3'. The primer sequences of glyceraldehyde-3-phosphate dehydrogenase (GAPDH), as a control, were 5'-TCGACAGTCAGCCGCATCTTCTTT-3' and 5'-ACCAAATCCGTTGACTCCGACCTT-3'. These primers were synthesized by the PrimerBank. To determine the reproducibility of the qRTPCR method for detecting the hsa_circ_0000520 levels in HCC tissue, we performed qRT-PCR three times to measure the differences between experimental batches. The data from qRT-PCR were analyzed by the $\Delta$ Ct method. All results were expressed as the mean $\pm \mathrm{SD}$. All assays were performed in a blinded fashion.

\section{Microarray data analysis}

The microarray detection was performed by Boao Bio-Tech (Beijing, China) under the guidance of the experiment work flow. The human $8 \times 15 \mathrm{~K}$ CIRCRNA Array was manufactured by Arraystar Technologies (Rockville, MD, USA). Overall, 65521 circRNAs were detected. Each circRNA was accurately identified using a specific probe targeting the circRNA-specific junction. Sample labeling, array hybridization, and raw data extraction were done with a high-quality control. Quantile normalization of raw data and subsequent data processing was performed using the $\mathrm{R}$ software package (R Version 3.1.2). 


\section{Statistical analyses}

All statistical analyses were performed by the Statistical Product and Service Solutions (SPSS) 22.0 software package (IBM Corporation, Armonk, NY, USA) and GraphPad Prism 6.0 (GraphPad Software, Inc., La Jolla, CA, USA). The differences in the levels of hsa_circ_0128298 between HCC and paired adjacent nontumorous tissues were assessed using the Student's $t$-test for paired data. Spearman's rank correlation coefficient was introduced to further calculate bivariate correlations. The receiver operating characteristic (ROC) curve was established to evaluate its diagnostic value. Independent prognostic factors for patient outcome were identified using the Cox regression model. The overall survival (OS) data were analyzed using the Kaplan-Meier method, and the differences were evaluated using log-rank tests. Two-sided $P$-values $<0.05$ were considered statistically significant.

\section{Results}

\section{Overview of circRNA profiles in HCC tissues}

Our microarray analysis revealed the circRNA expression profiles, as tested from three paired human HCC and paratumorous tissues. The detailed experimental process is shown in Figure 1, and clinicopathological characteristics are shown in Table 1. The median followup time was 37 months. The heat-map is depicted as a direct approach to visualize the distributions of the dataset for the circRNAs profiles (Figure 2A and B). The circRNA expression patterns between HCC and paratumorous tissues were found to be significantly different (Figure 2C). Differentially expressed circRNAs with statistical significance (fold changes $\geq 2.0$ and $P<0.05$ ) between groups were identified by volcano plot filtering (Figure 2D). After screening the differential circRNA expression profiles, we selected hsa_circ_0128298 and hsa_circ_0091582, the two most-changed circRNAs, for further study. We chose these two circRNAs for the following two reasons: 1) these two circRNAs had not been studied previously and 2) both circRNAs were in the top 10 highly expressed circRNAs.

\section{hsa_circ_0I 28298 was upregulated in HCC tissues}

qRT-PCR was used to detect the hsa_circ_0128298 and hsa_circ_0091582 expression levels in liver tissues from $\mathrm{HCC}$ patients. In the initial verification process with 30 pairs of HCC and paratumor tissues, hsa_circ_0091582 and hsa circ_0128298 were upregulated in HCC tissues $(P=0.023$ and 0.024 , respectively) (Figure 3 ). In the extended verification

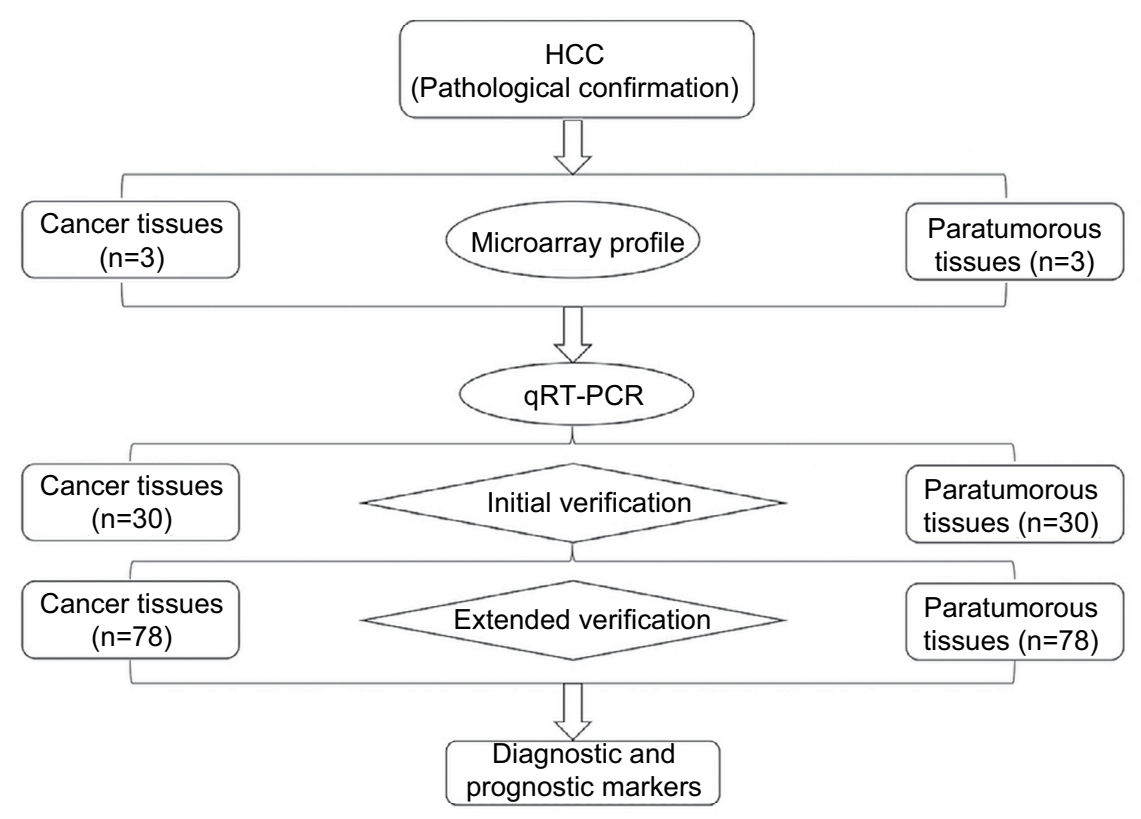

Figure I The detailed experimental process.

Notes: circRNA expression profiles were screened in three paired human HCC and paratumorous tissues. Targeted circRNAs were verified by qRT-PCR via initial verification in 30 pairs of samples and extended verification in 78 pairs of samples.

Abbreviations: circRNAs, circular RNAs; HCC, hepatocellular carcinoma; qRT-PCR, quantitative real-time polymerase chain reaction. 
Table I Clinicopathological characteristics of patient samples and expression of hsa_circ_0I28298 in hepatocellular carcinoma

\begin{tabular}{|c|c|}
\hline Variable & $\mathbf{n}$ \\
\hline \multicolumn{2}{|l|}{ Gender } \\
\hline$M / F$ & $70 / 8$ \\
\hline Age (years) & $37(4 I)$ \\
\hline \multicolumn{2}{|l|}{$\geq 55 /<55$} \\
\hline \multicolumn{2}{|c|}{ Vascular cancer embolus } \\
\hline Yes/no & $48 / 29$ \\
\hline \multicolumn{2}{|l|}{ HBSAG } \\
\hline Positive/negative & $65 / 13$ \\
\hline \multicolumn{2}{|l|}{ HBEAB } \\
\hline Positive/negative & $65 / 13$ \\
\hline \multicolumn{2}{|l|}{ Cirrhosis } \\
\hline Yes/no & $36 / 42$ \\
\hline \multicolumn{2}{|l|}{ AFP } \\
\hline$\geq 20 /<20$ & $52 / 26$ \\
\hline \multicolumn{2}{|c|}{ Intrahepatic metastasis } \\
\hline Yes/no & $23 / 55$ \\
\hline \multicolumn{2}{|l|}{ LN metastasis } \\
\hline Yes/no & $19 / 59$ \\
\hline \multicolumn{2}{|l|}{ Organ metastasis } \\
\hline Yes/no & $7 / 71$ \\
\hline \multicolumn{2}{|l|}{ Expression } \\
\hline Low/high & $39 / 39$ \\
\hline
\end{tabular}

Abbreviations: F, female; LN, lymph node; M, male; HBSAG, hepatitis Bs antigen; HBEAG, hepatitis Be antigen. process with 78 pairs of samples, only hsa_circ_0128298 was upregulated $(P=0.006)$ (Figures 4 and $\mathrm{S} 1)$.

\section{Upregulation of hsa_circ_0I28298 is associated with clinicopathological factors in HCC patients}

The data presented earlier demonstrated that the hsa circ_0128298 expression was significantly upregulated in HCC tissues; hence, we analyzed the association between its expression and clinicopathological factors of patients with HCC.

As shown in Table 2, in HCC tissues, a significant association was observed between the hsa_circ_0128298 expression and vascular cancer embolus $(P=0.012)$, lymphatic metastasis $(P=0.018)$, and organ metastasis $(P=0.048)$. However, no association was found between the hsa_circ_0128298 expression and other clinicopathological factors, including gender ( $P=0.455)$, age $(P=0.112)$, hepatitis Bs antigen $(P=0.615)$, hepatitis Be antigen $(P=0.761)$, cirrhosis $(P=0.65)$, AFP $(P=0.226)$, and intrahepatic metastasis $(P=0.11)$. The Spearman analysis in Table 3 indicated that the expression of
A
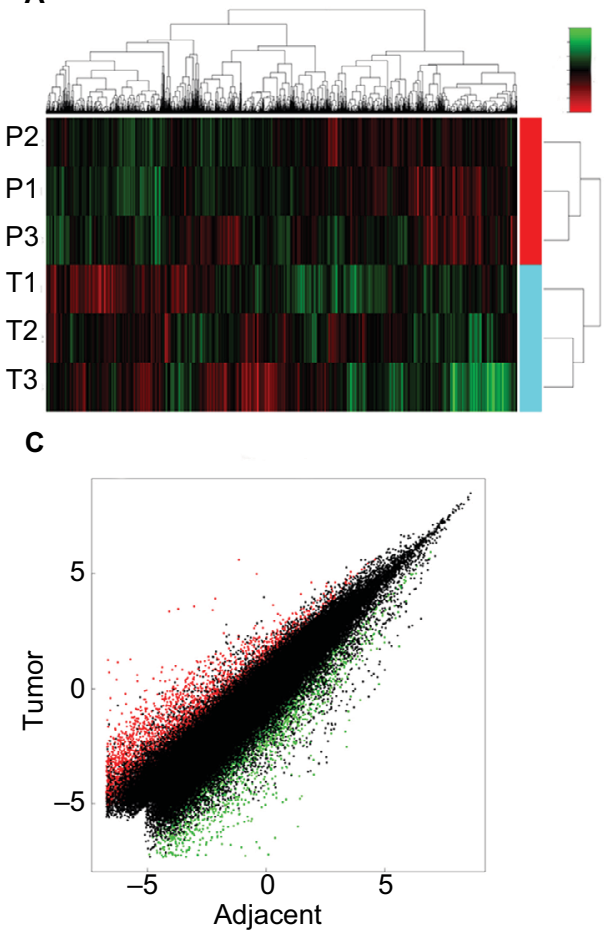

B

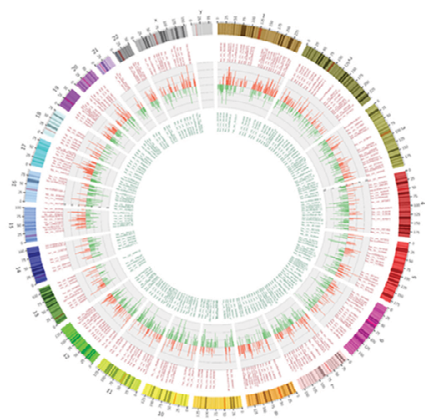

D

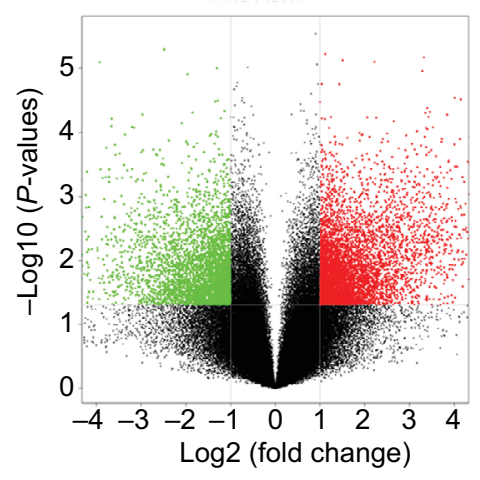

Figure 2 Overview of the microarray signatures.

Notes: (A) Unsupervised clustering analysis clearly distinguished the tumors and their corresponding nontumorous samples. (B) Illustration of the human genome showing the overall expression profile of the samples. (C) The whole transcriptome profiles of the tumor and their adjacent paratumor tissues were highly correlated. (D) Volcano plot showing the significantly deregulated genes in tumor samples. 
A

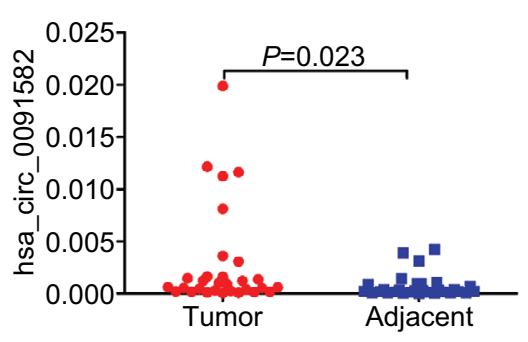

C

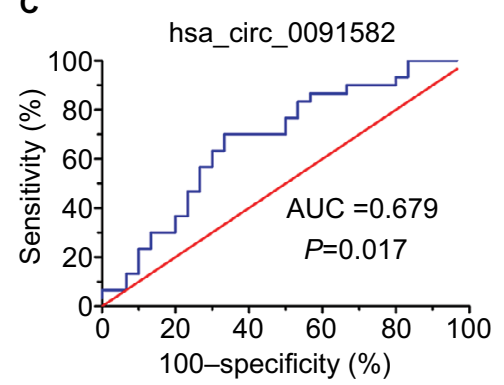

B

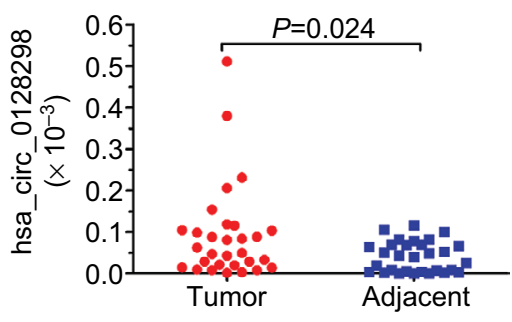

D

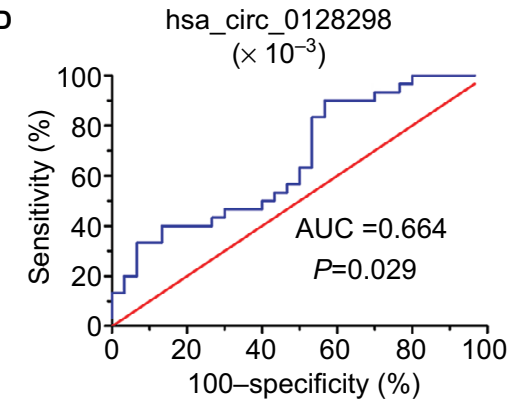

Figure 3 Target circRNAs from the initial verification from 30 pairs of samples.

Notes: (A and B) Increased expression of hsa_circ_0091582 and hsa_circ_0128298 in HCC tissues. RT-PCR was used to determine the expression levels. The $\triangle$ Ct values were determined by subtracting the $\mathrm{Ct}$ value for $\mathrm{GAPDH}$ from the $\mathrm{Ct}$ values for circRNAs. Larger $\Delta \mathrm{Ct}$ values indicate higher expression $(\mathrm{n}=30, P=0.023$ and $\mathrm{n}=30, P=0.024$, respectively). (C and D) The ROC curve for using hsa_circ_0091582 and hsa_circ_01 28298 as biomarkers $(P=0.017$ and 0.029 , respectively).

Abbreviations: circRNAs, circular RNAs; GAPDH, glyceraldehyde-3-phosphate dehydrogenase; HCC, hepatocellular carcinoma; ROC, receiver operating characteristics; RT-PCR, real-time polymerase chain reaction; AUC, area under the curve.

A

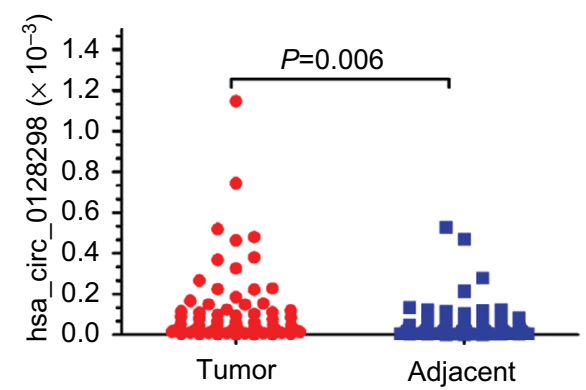

B

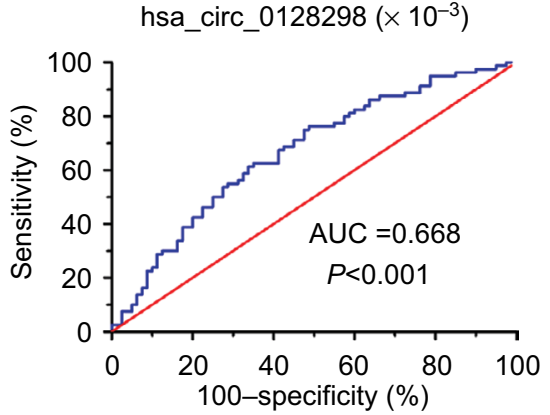

Figure 4 The expression levels and diagnostic value of hsa_circ_0I28298.

Notes: $(\mathbf{A})$ Increased expression of hsa_circ_0128298 in HCC tissues $(P=0.006)$. (B) The ROC curve of using hsa_circ_0128298 as a biomarker $(P<0.00 \mathrm{I}, \mathrm{AUC}=0.668)$.

Abbreviations: AUC, area under the curve; ROC, receiver operating characteristics.

hsa_circ_0128298 was correlated with intrahepatic metastasis $(P=0.021)$, lymphatic invasion $(P=0.004)$, and organ metastasis $(P=0.021)$.

\section{Potential diagnostic values of hsa circ_0I 28298}

The ROC curve was used to investigate the diagnostic value of hsa_circ_0128298 in distinguishing HCC tissues from paratumorous tissues. In the initial verification, we found that the proportion of hsa_circ_0091582 and hsa_circ_0128298 under the ROC curve (area under the curve [AUC]) was 0.679 and 0.664 ( $P=0.017$ and 0.029 , respectively) (Figure 3 ). In the extended verification, only hsa_circ_0128298 was con- firmed to have the diagnostic value of AUC $=0.668(P<0.001)$ (Figure 4A and B). The sensitivity and specificity were 0.674 and 0.805 , respectively.

\section{Potential prognostic values of hsa circ_0I 28298}

We analyzed all clinicopathological characteristics and hsa_circ_0128298 expression levels in the prognosis of HCC. Cox regression analysis was used to determine whether hsa circ_0128298 could serve as a prognostic factor. The univariate analysis showed that the gender $(P=0.018)$, AFP $(P=0.004)$, intrahepatic metastasis $(P=0.003)$, organ metastasis $(P=0.005)$, and hsa_circ_0128298 expression $(P=0.009)$ were independent 
Table 2 Correlation between hsa_circ_0128298 expression and clinicopathological characteristics of hepatocellular carcinoma patients

\begin{tabular}{|c|c|c|c|}
\hline Characteristics & $\begin{array}{l}\text { High } \\
\text { expression }\end{array}$ & $\begin{array}{l}\text { Low } \\
\text { expression }\end{array}$ & $P$-value \\
\hline \multicolumn{4}{|l|}{ Gender } \\
\hline$M / F$ & $34 / 5$ & $36 / 3$ & 0.455 \\
\hline \multicolumn{4}{|l|}{ Age (years) } \\
\hline$\geq 55 /<55$ & $22 / 17$ & $15 / 24$ & 0.112 \\
\hline \multicolumn{4}{|c|}{ Vascular cancer embolus } \\
\hline Yes/no & $29 / 9$ & $19 / 20$ & 0.012 \\
\hline \multicolumn{4}{|l|}{ HBSAG } \\
\hline Positive/negative & $29 / 10$ & $27 / 12$ & 0.615 \\
\hline \multicolumn{4}{|l|}{ HBEAB } \\
\hline Positive/negative & $32 / 7$ & $33 / 6$ & 0.761 \\
\hline \multicolumn{4}{|l|}{ Cirrhosis } \\
\hline Yes/no & $19 / 20$ & $17 / 22$ & 0.650 \\
\hline \multicolumn{4}{|l|}{ AFP } \\
\hline$\geq 20 /<20$ & 43343 & $26 / 13$ & 0.226 \\
\hline \multicolumn{4}{|c|}{ Intrahepatic metastasis } \\
\hline Yes/no & $15 / 24$ & $8 / 29$ & 0.110 \\
\hline \multicolumn{4}{|l|}{$\mathrm{LN}$ metastasis } \\
\hline Yes/no & $14 / 25$ & $5 / 34$ & 0.018 \\
\hline \multicolumn{4}{|l|}{ Organ metastasis } \\
\hline Yes/no & $6 / 33$ & $1 / 38$ & 0.048 \\
\hline
\end{tabular}

Abbreviations: $F$, female; LN, lymph node; $M$, male; HBSAG, hepatitis Bs antigen; HBEAG, hepatitis Be antigen.

Table 3 Spearman analysis of correlation between hsa circ_0I28298 and clinicopathological characteristics

\begin{tabular}{lll}
\hline Variables & \multicolumn{2}{l}{ hsa_circ_01 28298 expression level } \\
\cline { 2 - 3 } & Spearman correlation & P-value \\
\hline Gender & -0.101 & 0.07 \\
Age & 0.093 & 0.418 \\
Vascular cancer embolus & 0.143 & 0.216 \\
HBSAG & 0.149 & 0.165 \\
HBEAB & 0.055 & 0.632 \\
Cirrhosis & -0.084 & 0.466 \\
AFP & 0.058 & 0.617 \\
Intrahepatic metastasis & -0.244 & 0.034 \\
LN metastasis & -0.323 & 0.004 \\
Organ metastasis & -0.264 & 0.019 \\
\hline
\end{tabular}

Abbreviations: LN, lymph node; HBSAG, hepatitis Bs antigen; HBEAG, hepatitis Be antigen. factors (Table 4). Multivariate Cox regression analysis found that the AFP level $(P=0.018)$ and hsa_circ_0128298 expression level $(P=0.014)$ were the prognostic factors predicting poor survival among HCC patients (Table 4).

We further examined whether the hsa_circ_0128298 expression level was correlated with the outcome of $\mathrm{HCC}$ patients after hepatectomy. Kaplan-Meier's survival curves were used to compare the low $(n=39)$ and high $(n=39)$ hsa_circ_0128298 subgroups, and the results are presented in Figure 5. Patients with higher hsa_circ_0128298 expression levels had a statistically significant difference in the OS compared to the low expression group $(P=0.003)$.

\section{Discussion}

In recent years, there have been few advances in the treatment of HCC. Clinically, surgery and systemic chemotherapy are the standard treatments for HCC. ${ }^{3}$ However, only $13-15 \%$ of patients with HCC are suitable for surgery. Most patients are diagnosed with an advanced HCC, losing the precious opportunity for surgical operation. In addition, patients with HCC are prone to be multidrug resistant. ${ }^{3}$ There are several challenges in the diagnosis and treatment of HCC. First, there are difficulties in early diagnosis. Among the clinicopathological characteristics of HCC is its lack of specific early symptoms. Furthermore, the resection rate is only $15 \%$, but $\sim 50 \%$ patients are found to have distant metastases during treatment. ${ }^{14}$ Second, the heterogeneity of HCC further increases the difficulty. The genome-wide analysis of $\mathrm{HCC}$ has shown that genetic changes exist in $>10$ core signaling pathways. It has been reported that the alterations of multiple genes could possibly lead to ineffective treatments, resulting in poor prognosis. ${ }^{15}$ Therefore, the diagnosis and treatment of HCC should rely on early detection and diagnosis. The detection of HCC-related molecular markers predicting the early occurrence of $\mathrm{HCC}$ could be a more pragmatic way to diagnose and treat HCC.

Table 4 Univariate and multivariate analyses of various prognostic parameters in patients with HCC (Cox regression analysis)

\begin{tabular}{|c|c|c|c|c|c|c|}
\hline \multirow[t]{2}{*}{ Variables } & \multicolumn{3}{|c|}{ Univariate analysis } & \multicolumn{3}{|c|}{ Multivariate analysis } \\
\hline & $P$-value & Hazard ratio & $95 \% \mathrm{Cl}$ & $P$-value & Hazard ratio & $95 \% \mathrm{Cl}$ \\
\hline Gender & 0.018 & 3.268 & $1.220-8.750$ & & & \\
\hline Age & 0.944 & 1.001 & $0.972-1.03 \mid$ & & & \\
\hline Vascular cancer embolus & 0.08 & 0.513 & $0.243-1.084$ & & & \\
\hline HBSAG & 0.055 & 2.415 & $0.920-5.207$ & & & \\
\hline HBEAB & 0.312 & $1.51 \mathrm{I}$ & $0.679-3.364$ & & & \\
\hline Cirrhosis & 0.454 & 0.773 & $0.394-1.518$ & & & \\
\hline AFP & 0.004 & 4.25 & $1.420-4.329$ & 0.018 & 1.251 & $1.032-3.989$ \\
\hline Intrahepatic metastasis & 0.003 & 1.343 & I.144-7.056 & & & \\
\hline LN metastasis & 0.387 & 0.703 & $0.316-1.562$ & & & \\
\hline Organ metastasis & 0.005 & 0.244 & $0.091-0.657$ & & & \\
\hline hsa_circ_0128298 expression & 0.009 & 1.978 & $1.34 \mid-3.024$ & 0.014 & 6.661 & $2.66 \mid-8.418$ \\
\hline
\end{tabular}

Abbreviations: LN, lymph node; HBSAG, hepatitis Bs antigen; HBEAG, hepatitis Be antigen. 


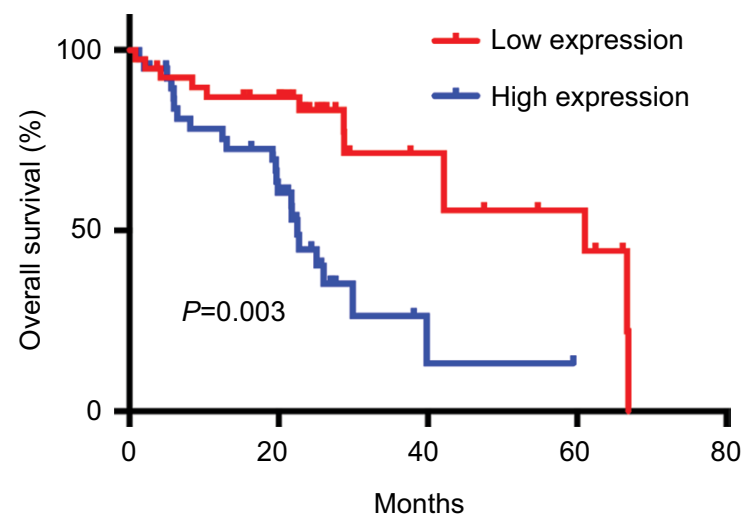

Figure 5 hsa_circ_0I 28298 can be an independent prognostic factor to predict OS. Note: Kaplan-Meier's analyses of correlations between the hsa_circ_0I28298 expression levels and OS of $78 \mathrm{HCC}$ patients are shown.

Abbreviations: HCC, hepatocellular carcinoma; OS, overall survival.

Increasing numbers of studies have shown that noncoding RNAs play an important role in the development of cancer. ${ }^{5-9,11}$ However, there are few studies on circRNA. circRNA is a type of special noncoding RNA and is the latest research hotspot in the RNA field. Unlike traditional linear RNAs (including 5' and 3' ends), circRNAs are closed-loop structures that are not affected by RNA exo-enzymes and are more stable and less degradable. ${ }^{7}$ Functionally, recent studies have shown that circRNAs are enriched at the miRNA-binding sites and play the role of miRNA sponges in cells, thus releasing the inhibitory effect of miRNAs on their target genes and increasing the target gene expression, which is known as the competitive endogenous RNA (ceRNA) mechanism. ${ }^{16,17}$ In addition, circRNAs play an important regulatory role in cancer via their interaction with cancer-associated miRNAs. ${ }^{18}$

Here, we identified a new circRNA (hsa_circ_0128298) that was significantly upregulated in HCC tissues. The expression of hsa_circ_0128298 was significantly associated with vascular cancer embolus, lymph node (LN), and organ metastasis $(P<0.05)$. The Spearman analysis of correlation between hsa_circ_0128298 and various clinicopathological factors indicated that the expression of hsa_circ_0128298 was correlated with intrahepatic metastasis, LN metastasis, and organ metastasis $(P<0.05)$. Moreover, we found that HCC patients with lower expression levels of hsa_circ_0128298 have prolonged OS compared to patients with higher hsa_circ_0128298 expression levels. The ROC analysis proved that hsa_circ_0128298 could be recognized as an HCC biomarker with favorable sensitivity and specificity. Note, in the Spearman analysis, hsa_circ_0128298 was negatively correlated with the three metastatic indicators, which is inconsistent with other results. Considering that hsa_circ_0128298 was an indicator of prognosis in HCC, we speculated that hsa_circ_0128298 did not affect the patients' prognosis by promoting metastasis. It might affect the prognosis of patients through other phenotypes, such as promoting vascular proliferation/immune escape. This also provides a certain direction for future research on the functional experiments of hsa_circ_0128298.

However, only 78 pairs of HCC tissues were analyzed in this study because of the limited number of available HCC samples. In addition, the tumor staging, $\mathrm{T}$ staging, and $\mathrm{N}$ staging were not included in our study due to a failure to acquire this information from some HCC patients. A larger number of samples should be tested at multiple centers to further confirm our conclusion. Furthermore, we did not test the molecular biological effects of hsa_circ_0128298 on HCC in vivo and in vitro.

\section{Conclusion}

Our data indicate that hsa_circ_0128298 expression is significantly upregulated in HCC samples and is a predictor of HCC occurrence and prognosis. Therefore, hsa_circ_0128298 could serve as a potential biomarker in the diagnosis of $\mathrm{HCC}$ and as a prognostic marker for the survival of $\mathrm{HCC}$ patients.

\section{Acknowledgments}

We thank the Innovation Project of Shandong Academy of Medical Science for support. This study was supported jointly by the National Natural Science Foundation of China (No 81603348), the China Postdoctoral fund (No 21300075311104), and the Shandong Postdoctoral Innovation special fund (No 201602012). The authors are grateful to the recruited patients and their kin.

\section{Disclosure}

The authors report no conflicts of interest in this work.

\section{References}

1. Jemal A, Bray F, Center MM, Ferlay J, Ward E, Forman D. Global cancer statistics. CA Cancer J Clin. 2011;61(2):69-90.

2. Thomas MB, Jaffe D, Choti MM, et al. Hepatocellular carcinoma: consensus recommendations of the National Cancer Institute Clinical Trials Planning Meeting. J Clin Oncol. 2010;28:3994-4005.

3. Takayama T. Surgical treatment for hepatocellular carcinoma. Jpn J Clin Oncol. 2011;41:447-454.

4. Fernandez M, Semela D, Bruix J, Colle I, Pinzani M, Bosch J. Angiogenesis in liver disease. J Hepatol. 2009;50:604-620.

5. Memczak S, Jens M, Elefsinioti A, et al. Circular RNAs are a large class of animal RNAs with regulatory potency. Nature. 2013;495(7441): 333-338.

6. Alhasan AA, Izuogu OG, Al-Balool HH, et al. Circular RNA enrichment in platelets is a signature of transcriptome degradation. Blood. 2016;127(9):e1-e11.

7. Zlotorynski E. Non-coding RNA: Circular RNAs promote transcription Nat Rev Mol Cell Biol. 2015;16(4):206. 
8. Szabo L, Morey R, Palpant NJ, et al. Statistically based splicing detection reveals neural enrichment and tissue-specific induction of circular RNA during human fetal development. Genome Biol. 2016;11(1):263.

9. Werfel S, Nothjunge S, Schwarzmayr T, Strom TM, Meitinger T, Engelhardt S. Characterization of circular RNAs in human, mouse and rat hearts. J Mol Cell Cardiol. 2016;98(1):103-107.

10. Yang D, Sun L, Li Z, Gao P. Noncoding RNAs in regulation of cancer metabolic reprogramming. Adv Exp Med Biol. 2016;927(1):191-215.

11. Hansen TB, Jensen TI, Clausen BH, et al. Natural RNA circles function as efficient microRNA sponges. Nature. 2013;495(7441):384-388.

12. Li Z, Huang C, Bao C, et al. Exon-intron circular RNAs regulate transcription in the nucleus. Nat Struct Mol Biol. 2015;22(3):256-264.

13. Granados-Riveron JT, Aquino-Jarquin G. e complexity of the translation ability of circRNAs. Biochim Biophys Acta. 2016;1859(10): 1245-1251.
14. Dulai PS, Singh S, Patel J, et al. Increased risk of mortality by brosis stage in non-alcoholic fatty liver disease: systematic review and metaanalysis. Hepatology. 2017;65:1557-1565.

15. Sun HZ, Song YL, Wang XY. Effects of different anesthetic methods on cellular immune and neuroendocrine functions in patients with hepatocellular carcinoma before and after surgery. J Clin Lab Anal. 2016;30:1175-1182.

16. Luan J, Jiao C, Kong W, et al. circHLA-C plays an important role in lupus nephritis by sponging miR-150. Mol Ther Nucleic Acids. 2018;10:245-253.

17. Li T, Mo X, Fu L, Xiao B, Guo J. Molecular mechanisms of long noncoding RNAs on gastric cancer. Oncotarget. 2016;7:8601-8612. doi: 10.18632/oncotarget.6926.

18. Chan JJ, Tay Y. Noncoding RNA:RNA regulatory networks in cancer. Int J Mol Sci. 2018;19(5). pii: E1310. 


\section{Supplementary material}

A

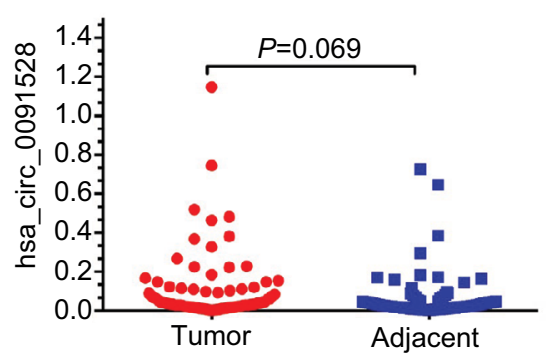

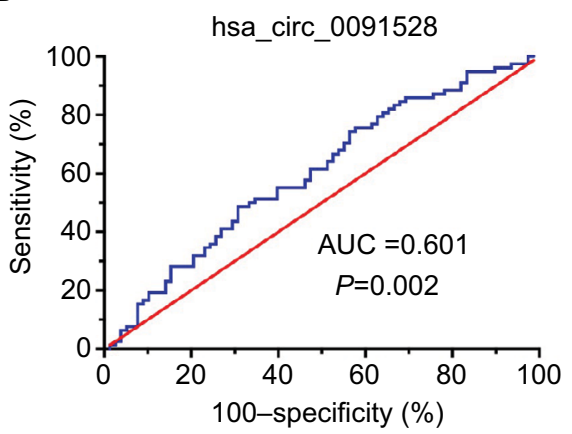

Figure SI The expression levels and diagnostic value of hsa_circ_0091528. Abbreviation: AUC, area under the curve.

Cancer Management and Research

\section{Publish your work in this journal}

Cancer Management and Research is an international, peer-reviewed open access journal focusing on cancer research and the optimal use of preventative and integrated treatment interventions to achieve improved outcomes, enhanced survival and quality of life for the cancer patient. The manuscript management system is completely online and includes

\section{Dovepress}

a very quick and fair peer-review system, which is all easy to use. Visit http://www.dovepress.com/testimonials.php to read real quotes from published authors. 